\title{
Effect of chronic and acute cigarette smoking on the pharyngo-upper oesophageal sphincter contractile reflex and reflexive pharyngeal swallow
}

\author{
K Dua, E Bardan, J Ren, Z Sui, R Shaker
}

\begin{abstract}
Background-Cigarette smoking is known to affect adversely the defence mechanisms against gastro-oesophageal reflux. The effect of smoking on the supraoesophageal reflexes that prevent aspiration of gastric contents has not been previously studied.

Aims-To elucidate the effect of cigarette smoking on two of the supraoesophageal reflexes: the pharyngo-upper oesophageal sphincter (UOS) contractile reflex; and the reflexive pharyngeal swallow.

Methods-Ten chronic smokers and 10 non-smokers were studied, before and $\mathbf{1 0}$ minutes after real or simulated smoking, respectively. UOS pressure and threshold volume for the reflexes were determined using a UOS sleeve assembly. Two modes of fluid delivery into the pharynx were tested: rapid injection and slow injection. Results-For both rapid and slow injections, the threshold volume for triggering the pharyngo-UOS contractile reflex was significantly higher in smokers than in non-smokers (rapid: smokers 0.42 (SE 0.07) $\mathrm{ml}$, non-smokers $0.16(0.04) \mathrm{ml}$; slow: smokers $0.86(0.06) \mathrm{ml}$, non-smokers $0.38(0.1) \mathrm{ml} ; \mathrm{p}<0.05)$. During rapid injection, the threshold volume for reflexive pharyngeal swallow was higher in smokers (smokers $0.94(0.09) \mathrm{ml}$, non-smokers 0.46 $(0.05) \mathrm{ml} ; \mathrm{p}<0.05)$. Acute smoking further increased the threshold volume for the pharyngo-UOS contractile reflex and reflexive pharyngeal swallow during rapid injection.
\end{abstract}

Conclusions-Smoking adversely affects stimulation of the pharyngo-UOS contractile reflex and pharyngeal reflexive swallow. These findings may have implications in the development of reflux related respiratory complications among smokers. (Gut 1998;43:537-541)

Keywords: smoking; supraoesophageal reflexes; pharyngo-upper oesophageal sphincter contractile reflex; reflexive pharyngeal swallow; airway protection; gastro-oesophageal reflux

A number of supraoesophageal reflexes have been proposed to help prevent aspiration of gastric contents. These reflexes enhance the physical barriers against entry of refluxate into the pharynx, such as the oesophago-upper oesophageal sphincter (UOS) and the pharyngo-UOS contractile reflexes ${ }^{1-4}$; close the introitus to the trachea, as with oesophagoglottal and pharyngoglottal closure reflexes ${ }^{5-7}$; or result in pharyngeal volume clearance as well as airway closure, as seen during reflexive pharyngeal swallow. ${ }^{8-11}$ Cigarette smoking is known to affect adversely the defence mechanisms against gastro-oesophageal reflux disease (GORD). ${ }^{12-21}$ However, its effect on the supraoesophageal reflexes described above has not been previously studied. Our aim in the present study was to elucidate the effect of chronic and acute smoking on two of these supraoesophageal reflexes: the pharyngo-UOS contractile reflex, and reflexive pharyngeal swallow.

\section{Methods}

We studied 10 healthy chronic smokers (mean age 34 (SD 5) years; six males) and 10 healthy non-smokers (mean age 33 (8) years; five males) in the sitting upright position. The protocol was approved by the Human Research Review Committee of the Medical College of Wisconsin, and study subjects gave informed consent. Smokers were defined as those with a history of smoking one or more packs of cigarettes per day for at least two years. Non-smokers were those who never smoked or occasionally smoked but had stopped over two years ago. Smokers were instructed to abstain from smoking for 12 hours prior to the study. We then studied them before and 10 minutes after smoking two cigarettes. The 10 minute interval after smoking was given to allow the pharyngeal temperature to return to baseline. Non-smokers were studied before and $10 \mathrm{~min}$ utes after simulated smoking of two unlit cigarettes. The duration of real/simulated smoking in each volunteer was kept to 15 minutes.

To monitor baseline UOS pressure and UOS response to pharyngeal water stimulation, and to determine the threshold volume for reflexive pharyngeal swallow, we adopted the method described previously. ${ }^{4}{ }^{10}$ We used an UOS sleeve assembly $(6 \times 0.6 \times 0.4 \mathrm{~cm}$; Dentsleeve, Adelaide, Australia) that had recording ports at the proximal and distal ends of the sleeve for manometric positioning. It also incorporated an injection port located $2 \mathrm{~cm}$ proximal to the sleeve device and two oesophageal ports located 5 and $7 \mathrm{~cm}$ apart. To prevent the possibility of anaesthetising the pharynx, the nasal passage was lubricated with a non-anaesthetic jelly (Surgilube, E. Fougera \& Co., Atlanta Inc., Melville, New York), applied with a cotton swab. The sleeve assembly was passed transnasally and positioned within the UOS such that
Accepted for publication 23 March 1998 


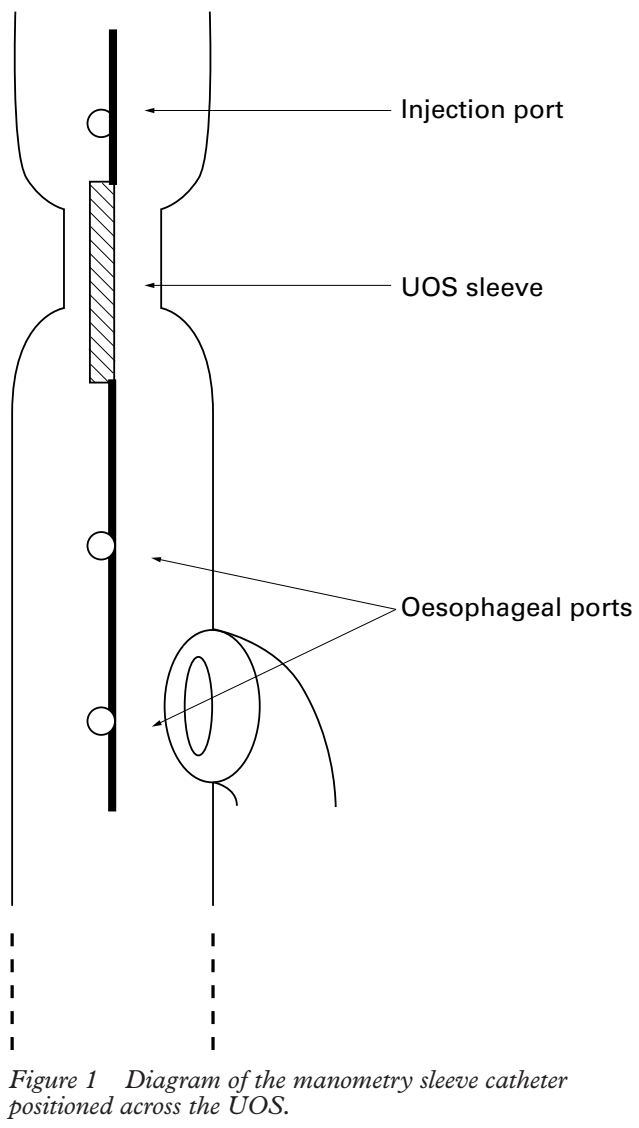

the injection port (the distal pharyngeal port, immediately above the sleeve segment) was oriented posteriorly and situated $2 \mathrm{~cm}$ above the UOS high pressure zone (fig 1). The pharyngeal, oesophageal, and sleeve ports were connected to pressure transducers in line with a minimally compliant pneumohydraulic system (Arndorfer Medical Specialties, Greendale, Wisconsin). With this arrangement, the onset and offset of water injection, UOS pressure, and oesophageal pressures were recorded on chart paper that was run at a speed of 25 $\mathrm{mm} / \mathrm{s}$. To avoid stimulation of swallowing, the pharyngeal ports (including top of the sleeve) were not perfused after the sleeve assembly was positioned. Studies were then performed following a 10 minute adaptation period. Rate of spontaneous swallowing per minute was then determined by a five minute observation period and this was repeated at the end of the study.

The UOS sleeve assembly was used in the present study for several reasons. The shape of the UOS sleeve conforms with that of the UOS opening. Once in position, the sleeve prevents axial rotation of the UOS catheter. As a result of this, the sleeve consistently measures the anterior-posterior pressure within the UOS and the pharyngeal water injection port is always maintained in the posterior orientation. Being $6 \mathrm{~cm}$ long, the sleeve can continuously record the UOS pressure, even during longitudinal UOS movement as observed with swallowing. Use of a side hole catheter may have given altered results for the above reasons and therefore was not used. The UOS sleeve assembly does have a slower response rate; in the present study, however, maximum rise in UOS pressure after pharyngeal water injection was measured and not the rate of rise.

For pharyngeal stimulation, graded volumes of water at room temperature were injected through the injection port $2 \mathrm{~cm}$ above the UOS. As gastro-oesophageal refluxate may enter the pharynx rapidly or may seep slowly through the UOS into the pharynx, ${ }^{22}$ we tested two modes of fluid delivery into the pharynx: rapid water injection and slow continuous water injection. Rapid water injection was performed by rapidly injecting water using a hand held syringe attached to the injection port. We started with $0.05 \mathrm{ml}$, followed by $0.1 \mathrm{ml}$ of water, and then increased the volume by $0.1 \mathrm{ml}$ increments until an irrepressible swallow occurred (reflexive pharyngeal swallow: rapid injection). Slow continuous perfusion was performed at a rate of $5.5 \mathrm{ml} / \mathrm{min}$ using a Harvard infusion pump (model N0975; Harvard Apparatus Co. Inc., Dover, Massachusetts) until an irrepressible swallow occurred (reflexive pharyngeal swallow: slow injection). Prior to each injection (rapid or slow), subjects were asked to swallow to clear the pharynx and then withhold swallowing until an irrepressible urge induced swallowing. Each injection was performed 10 seconds after the UOS stabilised at baseline and each volume was repeated three times.

The pharyngo-UOS contractile reflex was elicited based on the method described by Shaker et $a l .{ }^{4}$ After a 10 minute adaptation period, the consistent (three of three pharyngeal water injections) absolute rise in UOS pressure in $\mathrm{mm} \mathrm{Hg}$ above baseline was determined. The average end expiratory UOS pressure for a 10 second period before each injection was used as the baseline UOS pressure. Between injections, subjects were asked to swallow to clear the pharynx. We measured the maximum UOS pressure after pharyngeal water injection, excluding the three second interval before deglutitive relaxation, if a swallow occurred. This was done to avoid the commonly seen pressure increase that is registered by the sleeve during the orad excursion of the UOS immediately before its swallow induced relaxation.

Occurrence of swallow was documented by submental surface electromyography (EMG) of the mylohyoid/geniohyoid muscle group and characteristic UOS deglutitive relaxation; in addition, subjects signalled swallowing using a hand held marker that marked the chart paper when activated. All the above were concurrently recorded on the polygraph (Grass Instrument, Quincy, Massachusetts) chart paper.

In each subject, we determined, prior to and after smoking or simulated smoking: (1) the rate of spontaneous swallowing; (2) the smallest volume that consistently triggered the pharyngo-UOS contractile reflex on rapid and slow injections; (3) the latent period from onset of injection to onset of change in UOS pressure; (4) the percentage change in UOS pressure over preinjection baseline; and (5) the smallest volume that consistently triggered reflexive pharyngeal swallow on rapid and slow injections. 


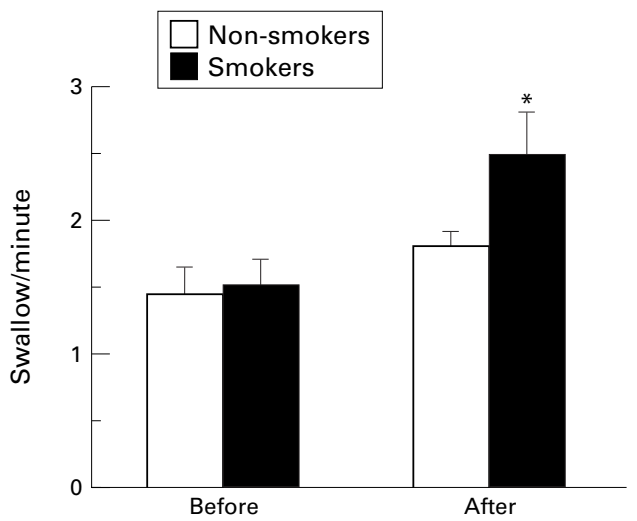

Figure 2 Number of spontaneous swallows per minute. ${ }^{*} p<0.05$.

Within and between group comparisons were done using paired and unpaired $t$ tests and analysis of variance. Bonferroni correction was applied for multiple comparisons. Values are given as mean (SE) unless stated otherwise.

\section{Results}

RATE OF SPONTANEOUS SWALLOWS

The rate of spontaneous swallows was similar between non-smokers and smokers $(1.4(0.2)$ and 1.5 (0.2) per minute respectively). However, real, but not simulated smoking of

A

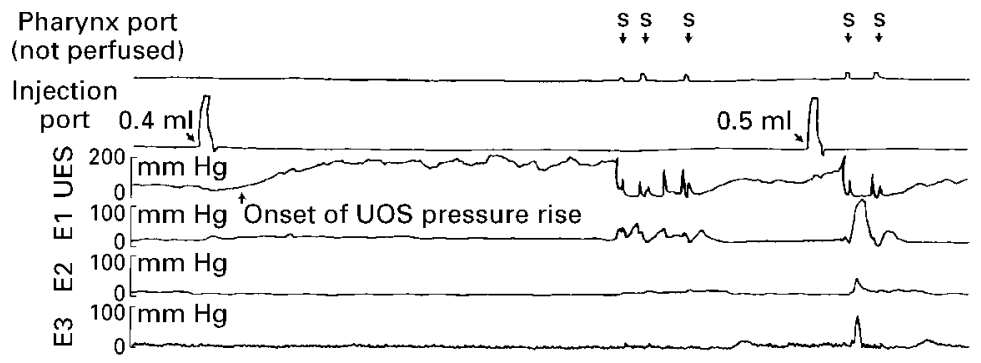

EMG

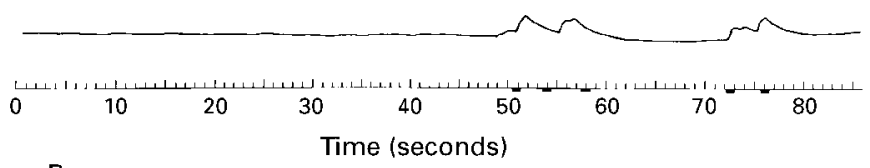

B

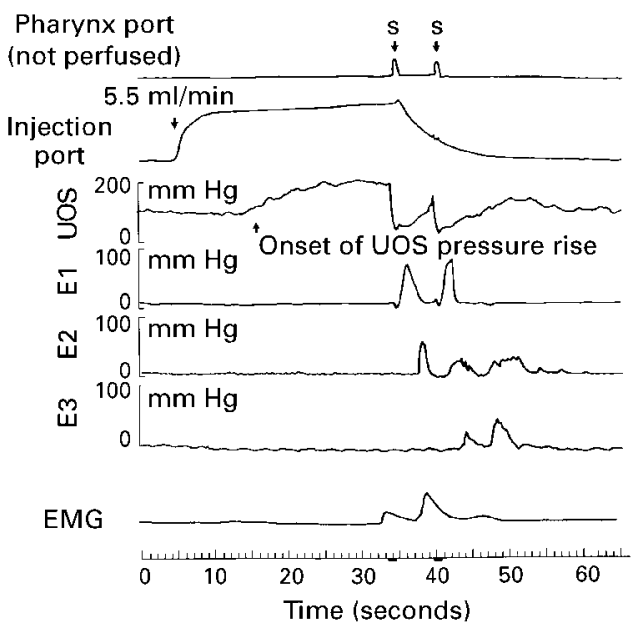

Figure 3 UOS pressure following rapid (A) and slow injection (B) of water into the pharynx. E1, E2, E3, proximal oesophageal perfusion ports; $S$, swallow. two cigarettes resulted in a significant increase in the spontaneous swallowing rate $(p<0.05$; fig 2).

PHARYNGO-UOS CONTRACTILE REFLEX

Except for one non-smoker and two smokers, at threshold volume, rapid or slow injection of water into the pharynx directed posteriorly resulted in an increase in UOS pressure (fig 3). Smoking of two cigarettes abolished the pharyngo-UOS contractile reflex in three additional smokers (one during rapid injection and two during slow injection). No similar effect was seen after simulated smoking of two unlit cigarettes by non-smokers. Results presented below exclude those in whom the pharyngo-UOS reflex could not be elicited and comparison is made between those in whom the reflex was triggered before and after smoking.

For both rapid and slow injections, the threshold volume required to trigger the pharyngo-UOS contractile reflex was significantly higher in smokers compared with nonsmokers $(p<0.05)$. Acute smoking of two cigarettes by smokers further increased the threshold volume required to trigger the reflex by rapid water injection (before smoking 0.42 (0.07), after smoking $0.68(0.09) \mathrm{ml} ; \mathrm{p}<0.05)$. For slow water injection, although the threshold volume further increased following smoking, the difference did not reach statistical significance (before smoking 0.87 (0.05), after smoking $1.0(0.13) \mathrm{ml}$; NS). Simulated smoking of two unlit cigarettes by nonsmokers did not significantly change the threshold volume for triggering the pharyngoUOS contractile reflex by either rapid or slow injections (rapid: before simulated smoking 0.16 (0.04), after simulated smoking 0.24 (0.07) $\mathrm{ml}$; slow: before simulated smoking 0.38 (0.1), after simulated smoking 0.38 (0.07) $\mathrm{ml}$; NS; fig 4).

The threshold volume of water required to trigger the pharyngo-UOS contractile reflex by slow water injection in both non-smokers and chronic smokers, was significantly higher compared with rapid water injection (non-smokers: rapid 0.16 (0.04), slow $0.38(0.1) \mathrm{ml}, \mathrm{p}<0.05$; smokers: rapid $0.42(0.07)$, slow $0.87(0.05)$ $\mathrm{ml}, \mathrm{p}<0.05$; fig 4 ).

In non-smokers, the time interval between the onset of water injection and the onset of change in UOS pressure (latent period) was 0.6 (0.07) seconds for rapid, and 6.4 (1.4) seconds for slow water injections. In smokers, although there was a trend for a longer interval period (rapid $1.4(0.2)$, slow 8.2 (1.8) seconds), this difference did not reach statistical significance when compared with nonsmokers. For both rapid and slow water injections, the latent period did not change significantly after simulated (in non-smoker group) or real (in smoker group) smoking. However, in both non-smokers and smokers, the latent period was significantly longer for slow injections compared with rapid injections $(\mathrm{p}<0.05)$.

The percentage rise over the basal UOS pressure following pharyngeal stimulation by 


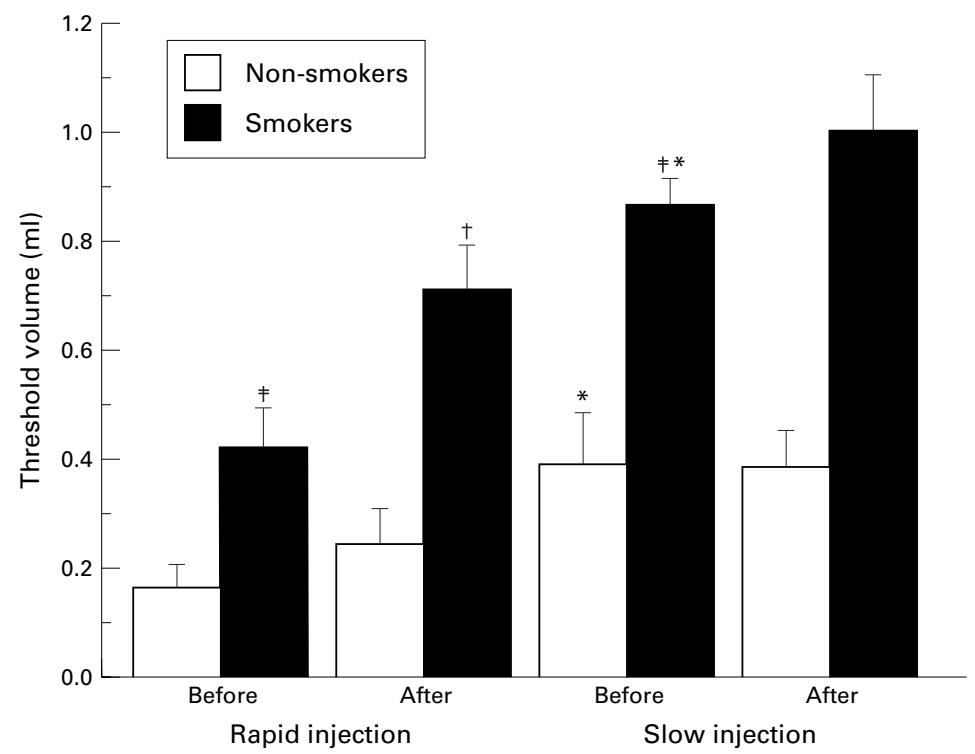

Figure 4 Threshold volume required for triggering the pharyngo-UOS contractile reflex. ${ }^{*} p<0.05$, slow versus rapid injection; $\neq p<0.05$, post-smoking versus pre-smoking; $\neq p<0.05$, smokers versus non-smokers.

both rapid and slow water injections was similar in smokers and non-smokers (smokers: rapid-before smoking 31 (13)\%, after smoking 44 (19)\%; slow-before smoking 31 (12)\%, after smoking 47 (14)\%; non-smokers: rapidbefore simulated smoking 60 (10)\%, after simulated smoking 51 (11)\%; slow-before simulated smoking $66(14) \%$, after simulated smoking $64(19) \%)$.

REFLEXIVE PHARYNGEAL SWALLOW

As shown in fig 5, in non-smokers and smokers, the threshold volume required to trigger reflexive pharyngeal swallow was significantly higher for slow water injection compared with rapid injection (non-smokers: rapid 0.46 (0.05), slow 1.1 (0.1) $\mathrm{ml}, \mathrm{p}<0.05$; smokers: rapid $0.94(0.09)$, slow $1.4(0.1) \mathrm{ml}, \mathrm{p}<0.05)$. For rapid water injection, this threshold

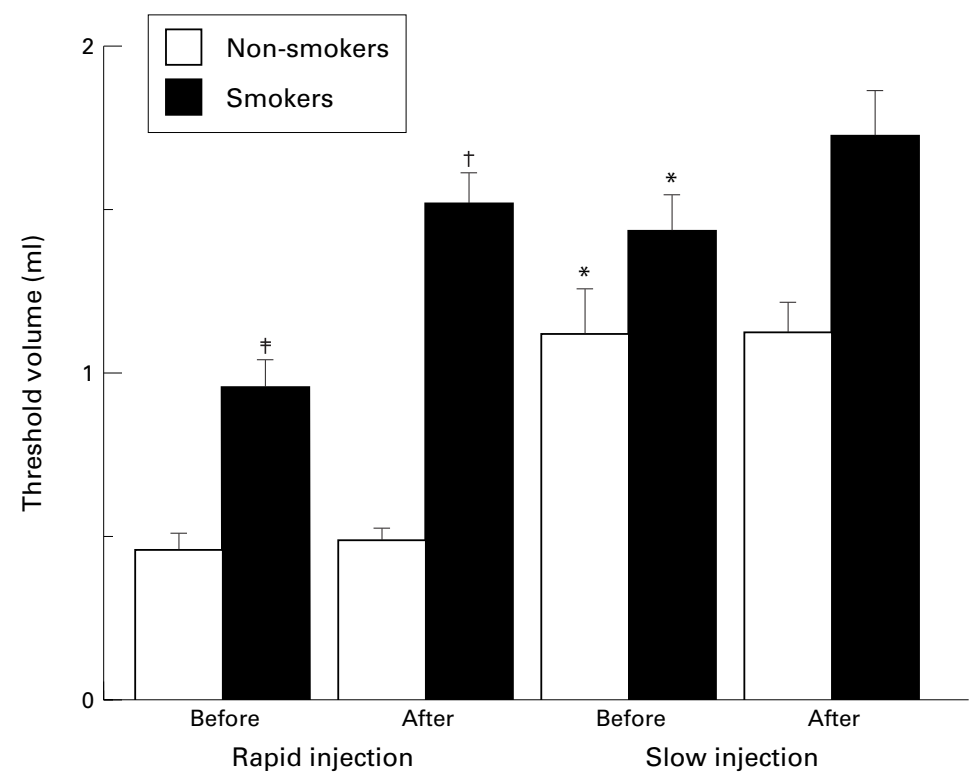

Figure 5 Threshold volume required for triggering reflexive pharyngeal swallow. ${ }^{\star} p<0.05$, slow versus rapid injection; $\neq p<0.05$, post-smoking versus pre-smoking; $\neq p<0.05$, smokers versus non-smokers. volume was significantly higher in smokers compared with non-smokers (smokers 0.94 (0.09), non-smokers $0.46(0.05) \mathrm{ml}, \mathrm{p}<0.05)$. After acute smoking of two cigarettes, the threshold volume further increased (after smoking $1.5(0.09) \mathrm{ml}, \mathrm{p}<0.05)$. A similar increase was not seen after simulated smoking by the non-smoker group (post-simulated smoking 0.48 (0.04) $\mathrm{ml}$, NS). However, contrary to rapid water injection, in both smokers and non-smokers, the threshold volume required to trigger reflexive pharyngeal swallow by slow pharyngeal water injection prior to real or simulated smoking was similar to that following real or simulated smoking respectively.

\section{Discussion}

In this study we determined the adverse effect of cigarette smoking on the pharyngo-UOS contractile reflex and reflexive pharyngeal swallow. The pharyngo-UOS contractile reflex and reflexive pharyngeal swallow are among a number of supraoesophageal reflexes that have been proposed to help prevent aspiration of gastric contents. These reflexes: enhance the physical barriers against entry of refluxate into the pharynx, namely the oesophago-UOS and pharyngo-UOS contractile reflexes ${ }^{1-4}$; close the introitus to the trachea, such as the oesophagoglottal and pharyngoglottal closure reflexes $^{5-7}$; or result in pharyngeal volume clearance as well as airway closure, such as reflexive pharyngeal swallow. ${ }^{8-11}$ The adverse effects of smoking on the defence mechanisms against gastro-oesophageal reflux such as lowering of upper and lower oesophageal sphincter pressures, ${ }^{14-18}$ increased frequency of failed secondary peristalsis, ${ }^{14}$ impaired oesophageal acid clearance, ${ }^{17} 1920$ and decrease in salivary base output, ${ }^{20}$ as well as its negative effect on gastric emptying ${ }^{12} 13$ have been described previously. Findings of the present study elucidate yet another area of the upper gastrointestinal tract on which cigarette smoking exerts a negative effect.

The mechanism responsible for these effects of smoking is currently not known. A significantly higher volume of water was required in chronic smokers to trigger the pharyngo-UOS contractile reflex and reflexive pharyngeal swallow compared with non-smokers. Furthermore, acute smoking of two cigarettes by smokers significantly increased the threshold volume required to trigger these reflexes during rapid water injection and abolished the pharyngo-UOS contractile reflex in one subject during rapid water injection and in two subjects during slow water injection. These negative effects of smoking may be due its effect on the sensory afferent branch of these reflexes. However, the possibilities of a central mechanism and/or a motor effect cannot be excluded. It is unlikely that pharyngeal temperature changes during smoking could have caused the above effect, as we allowed a 10 minute interval between completion of smoking and estimation of threshold volumes for the above reflexes. Similarly it is unlikely that the above effect could be secondary to the 
subject being more relaxed or adapted to the manometric catheter during the second half of the study compared with the first half, as no significant difference in the threshold volumes was noted before and after simulated smoking. It is possible that cigarette smoking may alter the concentration and/or function of the pharyngeal sensory nerve endings resulting in a higher threshold volume for pharyngo-UOS contractile reflex and reflexive pharyngeal swallow. Nicotine has been shown to affect adversely the oesophageal mucosa by producing free radicals, resulting in oxidative stress, ${ }^{23}$ and by inhibiting sodium transport. ${ }^{24}$ Cigarette smoking may have similar effects on the pharyngeal mucosa leading to alteration in the function of the pharyngeal sensory nerve endings.

In the present study, the frequency of spontaneous swallowing was similar between non-smokers and smokers. Acute real but not simulated smoking significantly increased the frequency of spontaneous swallowing. Earlier studies have suggested that smoking may decrease salivary output. $^{20}$ Although oral manipulation inherent with cigarette smoking can cause hypersalivation, it is unlikely that excessive salivation accounted for the increased frequency of spontaneous swallowing as this was not observed during simulated oral manipulation of unlit cigarettes by non-smokers. Furthermore, swallow frequency was measured before and after completion of smoking and not during the act of smoking. It is possible that the "irritant" effect of cigarette smoke on the oral/ pharyngeal mucosa may have been responsible for the observed increase in the frequency of spontaneous swallowing, or, alternatively, this could be secondary to a central effect of nicotine.

In summary, in chronic smokers the threshold volume for triggering the pharyngo-UOS contractile reflex and reflexive pharyngeal swallow is significantly higher compared with that in non-smokers. Acute smoking of cigarettes further affects these reflexes adversely. These findings identify yet another deleterious effect of cigarette smoking that can further weaken the airway protective mechanisms against aspiration. These findings may have implications in the pathogenesis of reflux related respiratory complications among smokers.
This work was supported in part by NIH grant no. RO1-DC00669, Merit Review Grant from the Department of the Medical College of Wisconsin. Part of this work was presented at the Research Forum during Digestive Disease Week in May 1995 and published in abstract form (Gastroenterology 1995;108:A594).

1 Reynolds RPE, Effer GW, Bendeck MP. The upper esophageal sphincter in the cat: the role of central innervation
assessed by transient vagal blockade. Can $\mathcal{F}$ Physiol assessed by transient

2 Medda BK, Lang IM, Layman RD, et al. Characterization and quantification of a pharyngo-UES contractile reflex in the cat. Am $\mathcal{F}$ Physiol 1994;267:G972-83.

3 Ren J, Shaker R, Lang I, et al. Effect of volume, temperature and anesthesia on the pharyngo-UES contractile reflex in humans [abstract]. Gastroenterology 1995;108:A677.

4 Shaker R, Ren J, Xie P, et al. Characterization of the pharyngo-UES contractile reflex in humans. Am $\mathcal{F}$ Physiol 1997;36:G854-8.

5 Shaker R, Dodds WJ, Ren J, et al. Esophagoglottal closure reflex: a mechanism of airway protection. Gastroenterology 1992;102:857-61.

6 Shaker R, Ren J, Medda B, et al. Identification and characterization of the esophagoglottal closure reflex in a feline model. Am F Physiol 1994;266:G147-53.

7 Ren J, Shaker R, Dua K, et al. Glottal adduction response to pharyngeal water stimulation: evidence for a pharyngoglottal closure reflex [abstract]. Gastroenterology 1994;106: tal closu.

8 Nishino T, Takizawa K, Yokokawa N, et al. Depression of the swallowing reflex during sedation and/or relative analgesia produced by inhalation of $50 \%$ nitrous oxide in oxygen. Anesthesiology 1987;67:995-8.

9 Nishino T. Swallowing as a protective reflex for the upper respiratory tract. Anesthesiology 1993;79:588-601.

0 Shaker R, Ren J, Zamir Z, et al. Effect of aging, position, and temperature on the threshold volume triggering pharyngeal swallows. Gastroenterology 1994;107:396-402.

11 Trifan A, Shaker R, Ren J, et al. Inhibition of resting lower esophageal sphincter pressure by pharyngeal water stimulation in humans. Gastroenterology 1995;108:441-6.

12 Scott AM, Kellow JE, Shuter B, et al. Effect of cigarette smoking on solid and liquid intragastric distribution and gastric emptying. Gastroenterology 1993;104:410-16.

gastric emptying. Gastroenterology $1993 ; 104: 410-16$.
Johnson RD, Horowitz M, Maddox AF, et al. Cigarette smoking and rate of gastric emptying: effect on alcohol smoking and rate of gastric emptyit
absorption. BM7 1991;302:20-3.

14 Dua K, Bardan E, Xie P, et al. Effect of chronic and acute cigarette smoking on upper and lower esophageal sphincters and on secondary esophageal peristalsis. Gastroenterology 1996;110:A658.

15 Kahrilas PJ, Gupta RR. Mechanisms of acid reflux associated with cigarette smoking. Gut 1990;31:4-10.

16 Chattopadhyay DK, Greaney MG, Irvin TT. Effect of cigarette smoking on the lower oesophageal sphincter. Gut 1977;18:833-5.

17 Stanciu C, Bennett JR. Smoking and gastro-oesophageal reflux. BMF 1972;3:793-5.

18 Dennish GW, Castell DO. Inhibitory effect of smoking on the lower esophageal sphincter. N Engl f Med 1971;284: $1136-7$.

19 Rahal PS, Wright RA. Transdermal nicotine and gastroesophageal reflux. Am f Gastroenterol 1995;90:919-21.

20 Kahrilas PJ, Gupta RR. The effect of cigarette smoking on salivation and esophageal acid clearance. $\mathcal{F}$ Lab Clin Med 1989;114:431-8.

21 Rattan S, Goyal RK. Effect of nicotine on the lower esophageal sphincter. Gastroenterology 1975;69:154-9.

22 Shaker R, Dodds WJ, Hogan WJ, et al. Mechanisms of esophago-pharyngeal acid regurgitation [abstract]. Gastroenterology 1991;100:A494.

23 Wetscher GJ, Bagchi D, Perdikis G, et al. In vitro free radical production in rat esophageal mucosa induced by nicotine. Dig Dis Sci 1995;40:853-8.

24 Orlando RC, Bryson JC, Powell DW. Effect of cigarette smoke on esophageal epithelium of the rabbit. Gastroenterology 1986;91:1536-42. 